\title{
Top quark properties at the Tevatron
}

\author{
Arán García-Bellido ${ }^{1}$ \\ University of Washington, Seattle WA 98195, USA \\ FERMILAB-CONF-04-320-E
}

\begin{abstract}
Recent preliminary results on top quark properties from the CDF and D $\varnothing$ collaborations at the Fermilab Tevatron are described. These analyses include: measurements of the ratio $B(t \rightarrow$ $W b) / B(t \rightarrow W q)$ and of $B(t \rightarrow \tau v q)$, search for anomalous kinematics, studies on the helicity of $W$ bosons originating from top quark decays, spin correlations between top quark pairs, and searches for $t \bar{t}$ resonance production in models beyond the standard model.
\end{abstract}

\section{INTRODUCTION}

The top quark has offered a new window to look into standard model physics and beyond since its discovery at the Fermilab Tevatron proton-antiproton collider [1]. With a mass of $178 \pm 4 \mathrm{GeV} / c^{2}$ and a width of $1.5 \mathrm{GeV}$ [2] the top quark is unique in the quark sector. Its large mass, of the order of the Higgs vacuum expectation value, may indicate its special role in electroweak symmetry breaking and hint to a connection with new physics which could couple to the top quark easier than to other fermions; its large width, larger than $\Lambda_{\mathrm{QCD}} \sim 200 \mathrm{MeV}$, means it decays before hadronization occurs and thus the decay products conserve the angular properties of the parent top quark. The study of top quark properties is yet an open field with many unknown quantities such as its charge, spin or coupling strength, that can bring great insight into some of the remaining questions in our field.

Top quarks are predominantly produced in pairs at the Tevatron with a cross section of around $7 \mathrm{pb}$ [3]. With an increased center-of-mass energy of $1.96 \mathrm{TeV}$ for Run II, new detectors and analyzed luminosities of $110-190 \mathrm{pb}^{-1}$, the Tevatron collaborations now have surpassed the Run I collection of top quark candidates. This new sample has been studied to understand the details of top quark decay properties, and look for signs of new physics. The final state of $t \bar{t}$ pair production is determined by $t \rightarrow W b$, which is the standard model dominant decay for top quarks, and referred to as dilepton, lepton plus jets, and all hadronic, depending on whether both $W$ bosons decay leptonically (to $\ell v$ ), one decays leptonically and the other hadronically (to $q \bar{q}^{\prime}$ ) or both decay hadronically.

This letter is divided as follows: the CDF analysis on the branching ratio $R=B(t \rightarrow$ $W b) / B(t \rightarrow W q)$ is described as a test of the unitarity of CKM matrix; in the next section, anomalous couplings of top quarks are tested by measuring the ratio of the cross section to dileptons over the cross section to lepton plus jets; another search for new physics is performed in the analysis of top quark decays to $\tau \nu b$ which rate can be enhanced in some

\footnotetext{
${ }^{1}$ On behalf of the CDF and DØ collaborations
} 
models of supersymmetry; the next section describes detailed studies of the kinematic distributions of top events to detect any non-standard model features, like increased top $p_{\mathrm{T}}$ spectra; the following section deals with the measurement of the $W$ helicity as derived from the lepton $p_{\mathrm{T}}$ spectra; and finally, a measurement of the spin correlation between top quarks and a search for $t \bar{t}$ resonances with Run I data are briefly reviewed.

\section{MEASUREMENT OF $B(t \rightarrow W b) / B(t \rightarrow W q)$}

The decay of top quarks is expected to be around $100 \%$ to a $\mathrm{W}$ boson and a $b$ quark in the framework of the standard model. To measure any deviation from the standard model, the ratio $R=B(t \rightarrow W b) / B(t \rightarrow W q)$ can be formed, where $q$ may be any $-1 / 3$ charged quark. In the standard model we can describe this ratio as a function of the CKM matrix elements:

$$
R=\frac{\left|V_{t b}\right|^{2}}{\left|V_{t d}\right|^{2}+\left|V_{t s}\right|^{2}+\left|V_{t b}\right|^{2}}=\left|V_{t b}\right|^{2}
$$

where the second equality is derived assuming the unitarity of the CKM matrix. The current PDG estimate of $\left|V_{t b}\right|>0.999$ at the $90 \%$ confidence level means that we should expect $R>0.998$. But this is only if unitarity holds. For example, a fourth generation of heavy quarks could exist which would break unitarity driving $R$ away from unity.

Both CDF and DØ have performed analyses to measure $R$, here we describe the CDF analysis [4]. $R$ can be determined by the relative rates of identified $b$ quark jets in $t \bar{t}$ events. The two top quarks have each a probability $R$ to decay to a $b$ quark, by counting the number of events with zero, one and two $b$-tagged jets $\left(N_{0}, N_{1}\right.$ and $N_{2}$ respectively) we can obtain $R \varepsilon_{b}$, where $\varepsilon_{b}$ is the efficiency to identify ("tag") the $b$ quark jet:

$$
\begin{gathered}
N_{0} \propto\left(1-R \varepsilon_{b}\right)^{2}, N_{1} \propto 2 R \varepsilon_{b}\left(1-R \varepsilon_{b}\right), N_{2} \propto\left(R \varepsilon_{b}\right)^{2} \Rightarrow \\
R \varepsilon_{b} \propto \frac{2}{N_{1} / N_{2}+2} \propto \frac{1}{2 N_{0} / N_{1}+1} \propto \frac{1}{\sqrt{N_{0} / N_{2}}+1}
\end{gathered}
$$

$R$ can be extracted by measuring $\varepsilon_{b}$ independently, from $t \bar{t}$ simulation calibrated with complimentary data samples. $b$ jets are identified using the SECVTX algorithm, which searches for displaced vertices in jets.

The data analyzed corresponds to $161 \mathrm{pb}^{-1}$ and is divided into three $t \bar{t}$ enriched samples: dilepton, lepton plus three jets and lepton plus four jets, according to whether the two $W$ bosons decay to leptons only or quarks and leptons and the event has exactly three or more than three reconstructed jets. The analysis proceeds as follows: in samples selected for $t \bar{t}$, the expected background and observed data events are divided as a function of the number of $b$-tagged jets. The expected number of events from signal $t \bar{t}\left(N_{0}, N_{1}\right.$ and $\left.N_{2}\right)$, are then used in a likelihood, together with the background and observed data, to give the best estimate of $R \varepsilon_{b}$ and a lower limit on $R$.

Table 1 shows the expected number of background events and the observed data as a function of the number of $b$-tagged jets when the $t \bar{t}$ selection is applied.

The definition of $\varepsilon_{b}$ needs to be corrected when we allow for top quarks to decay to other non- $b$ ("light") quarks: $t \rightarrow W q$, as we do in this analysis. To account for this 
TABLE 1. The number of observed events and expected background for the three data samples dilepton (LL), lepton plus three jets (L3J) and lepton plus more than three jets (L4J) divided into the number of observed $b$ jets

\begin{tabular}{lcccccc}
\hline & \multicolumn{3}{c}{ Data } & \multicolumn{3}{c}{ Background } \\
sample & LL & L3J & L4J & LL & L3J & L4J \\
\hline 0-tag & 5 & 358 & 79 & $2.0 \pm 0.6$ & - & - \\
1-tag & 4 & 26 & 23 & $0.2 \pm 0.2$ & $16.0 \pm 2.4$ & $4.2 \pm 0.7$ \\
2-tag & 3 & 3 & 5 & 0 & $0.8 \pm 0.2$ & $0.2 \pm 0.1$ \\
\hline
\end{tabular}
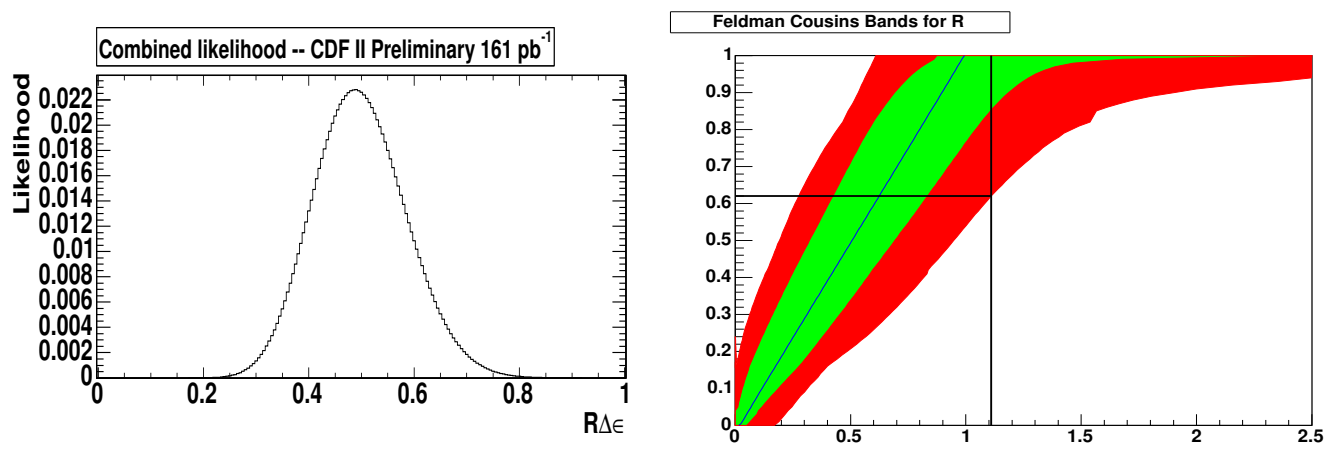

FIGURE 1. The combined likelihood function as a function of $R \Delta \varepsilon$ (left). On the right, the plane $R_{\text {true }} v s . R_{\text {measured }}$ in the Feldman-Cousins analysis, with $95 \%$ (red) and $68 \%$ (green) confidence bands taking into account both statistical and systematic uncertainties.

we calculate the efficiency to tag a $q$-type jet as $\varepsilon_{q}$, which is generally small, and then redefine the efficiency to tag a $b$ jet as: $R \Delta \varepsilon+\varepsilon_{q}$, where $\Delta \varepsilon \equiv \varepsilon_{b}-\varepsilon_{q}$. From $t \bar{t}$ Monte Carlo samples, $\Delta \varepsilon=0.436 \pm 0.032$, where the uncertainty is calculated using calibration data and simulated samples.

A likelihood function is built as the product of the Poisson probabilities to observe zero, one and two $b$-tagged jets for the expected number of events with the same number of tags: $\mathscr{L}=\prod_{n=0,1,2} \mathscr{P}\left(N_{n}^{\text {observed }}, N_{n}^{\text {expected }}\right)$. This function is maximal for that value of $R \Delta \varepsilon$ that is most consistent with data. The maximum of the likelihood curve is found to be at: $R \Delta \varepsilon=0.49_{-0.08}^{+0.09}$, see Fig. 1 . If we plug in the measured value of $\Delta \varepsilon=0.436 \pm 0.032$, we obtain a measurement of $R$ consistent with the standard model within errors: $R=1.11_{-0.19}^{+0.21}$. To set a limit on $R$, a Feldman-Cousins [5] prescription is applied to find at 95\% C.L. that $R>0.62$ (Fig. 1). This improves the CDF Run I limit [6] of $R>0.56$.

\section{RATIO OF DILEPTON TO $\ell+J E T S$ CROSS SECTIONS}

The cross section for $t \bar{t} \rightarrow \ell \ell+$ jets and $t \bar{t} \rightarrow \ell+$ jets should be identical since both final states arise from the standard model decay of top quarks which is almost $100 \%$ to $\mathrm{Wb}$. By measuring the ratio of dilepton to $\ell+$ jets final state cross sections, we are testing that 
in both analysis we are dealing with the same kind of top quark decays. Furthermore, the ratio cancels systematic uncertainities correlated between the two analyses and makes it independent of the theoretical cross section value, which depends on parton density functions and the top quark mass, etc. The final result from CDF, after building a probability distribution for $R_{\sigma}=\frac{\sigma_{\ell \ell}}{\sigma_{\ell+\text { jets }}}$ with the dilepton data of $126 \mathrm{pb}^{-1}$ and $\ell+$ jets data of $108 \mathrm{pb}^{-1}$, yields: $R_{\sigma}=1.45_{-0.55}^{+0.83}$ [3]. This is in agreement with the standard model prediction of $R_{\sigma}=1$. A 95\% C.L. can be set between: $0.46<R_{\sigma}<4.45$. Any deviation from unity would imply the presence of non-standard model decays of top $(t \rightarrow X b)$.

\section{RATE OF TOP QUARK DECAYS TO $\tau v q$}

Analyses involving $\tau$ leptons at the Tevatron are challenging because they require very good calorimetry and track isolation techniques. Usually, $t \bar{t}$ analyses are based on the leptonic decays of the $W$ boson to an electron or a muon. However, $t \bar{t}$ events can be identified by requiring that one of the $W$ bosons decays to an electron or a muon, and the other to a tau, which then decays hadronically. Although leptonic decays of taus account for $35 \%$ of all tau decays, it is difficult to differentiate them from prompt leptons, so present analyses at the Tevatron look for one or three prong hadronic decays of taus. We expect the $W$ boson to decay equally to all three families of leptons, but in $t \bar{t}$ events, given the high top mass, new physics in the form of $t \rightarrow H^{ \pm} b \rightarrow \tau^{ \pm} v b$, where $H^{ \pm}$is the charged Higgs, could appear and produce an anomalous rate of $t \bar{t}$ events with taus in the final state. For example, in the minimal supersymmetric extension of the standard model (MSSM) with high $\tan \beta$ values, the decay $t \rightarrow H b$ is expected to dominate over the standard model one $t \rightarrow W b$. CDF has looked for any such anomalies in $193 \mathrm{pb}^{-1}$ of Run II data by measuring the ratio of the observed rate to the standard model rate [7]:

$$
r_{\tau}=\frac{B^{\text {observed }}(t \rightarrow b \tau v)}{B^{\operatorname{SM}}(t \rightarrow b \tau v)}
$$

This analysis requires an isolated electron or muon with $p_{\mathrm{T}}>20 \mathrm{GeV} / c$, an identified tau lepton with $p_{\mathrm{T}}>15 \mathrm{GeV} / c$ and two jets. Tau identification is of course crucial to this analysis. The efficiency to identify taus is measured in $W \rightarrow \tau v$ Monte Carlo and then scaled by comparing it to an independent data sample dominated by $W \rightarrow \tau \nu$. Figure 2 displays the agreement between Monte Carlo and data for the tau identification efficiency.

The principal background is $W+$ jets where a jet is misidentified as a tau. $Z \rightarrow \tau \tau+$ jets is also an important background because it contains real taus, but cuts on the total transverse energy of the event $H_{T}>205 \mathrm{GeV}$ and a $Z$ mass window cut, can effectively reduce this background.

Finally, two events are observed and 2.3 expected in the electron-tau channel, and zero observed and 1.00 expected in the muon-tau channel. This allows to set an upper limit of $r_{\tau}<5.0$ at $95 \%$ C.L. which is consistent with the standard model prediction of $r_{\tau}=1$. A better tau identification and a larger data sample will improve this constraint on $r_{\tau}$. 

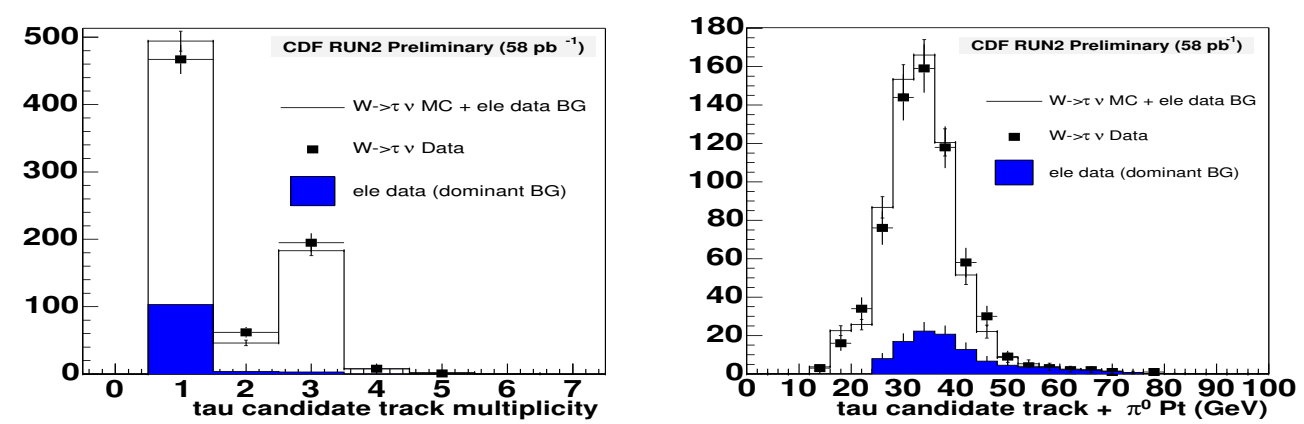

FIGURE 2. The number of tracks in taus (left) and tau $p_{\mathrm{T}}$ (right) distributions for $W \rightarrow \tau v$ data and $W \rightarrow \tau \nu$ Monte Carlo plus electron data background. The $W \rightarrow \tau v$ data is an independent sample used to estimate the agreement between data and Monte Carlo in the tau identification efficiency

\section{SEARCH FOR ANOMALOUS KINEMATICS}

The Run I cross section measurement in the dilepton channel [8] showed in the CDF data some peculiar candidate events, with large $E_{\mathrm{T}}$ and lepton $p_{\mathrm{T}}$, that did not resemble the expected kinematics from standard model top quark decays. CDF has performed a new search with $193 \mathrm{pb}^{-1}$ of Run II data to look for such events with anomalous kinematics in the top dilepton sample [9]. There are models of physics beyond the standard model that can explain this apparent discrepancy, for example as decays of heavy squarks [10]. The analysis is based on the comparison of kinematic features observed in the data with those expected from the standard model, to isolate that subsample of events that departs the most from expectation and to assign a probability to quantify that departure.

The event selection is the same used for the dilepton $t \bar{t}$ cross section measurement [11], benefiting from a high purity of the selected candidates, albeit its small acceptance. This leaves 13 candidate events with $10.2 \pm 1.4$ expected.

A few specific kinematic variables have been selected to discriminate between new physics signals and standard model processes. Using the Run I events as a guide, the leading lepton $p_{\mathrm{T}}$ and the missing energy $E_{\mathrm{T}}$ are expected to be sensitive to new heavy particles decaying in the detector. Also, the angular distribution of these possible new decays would exhibit a more back-to-back signature, whereas top quark decays tend to be more spherical. Therefore, the angle between the leading lepton and the $\mathscr{E}_{\mathrm{T}}$ in the plane transverse to the beam $\Phi_{l m}$ is used to detect the presence of many possible different final state kinematics produced by new physics. Finally, a topological variable based on the top dilepton kinematics is introduced to directly measure how compatible the event is with a $t \bar{t}$ dilepton event based on the predicted and measured values of the $B_{\mathrm{T}}$ :

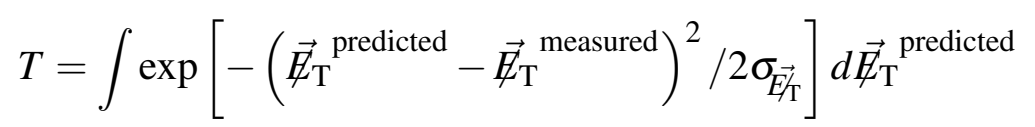

A new technique has been devised to loop over the candidate events creating subsamples of events to which an event weight is assigned based on the probability for that event to have the observed values of the four variables (leading lepton $p_{\mathrm{T}}, Z_{\mathrm{T}}, \Phi_{l m}$ and $T$ ), given the expected standard model distributions. This produces several subsets of 

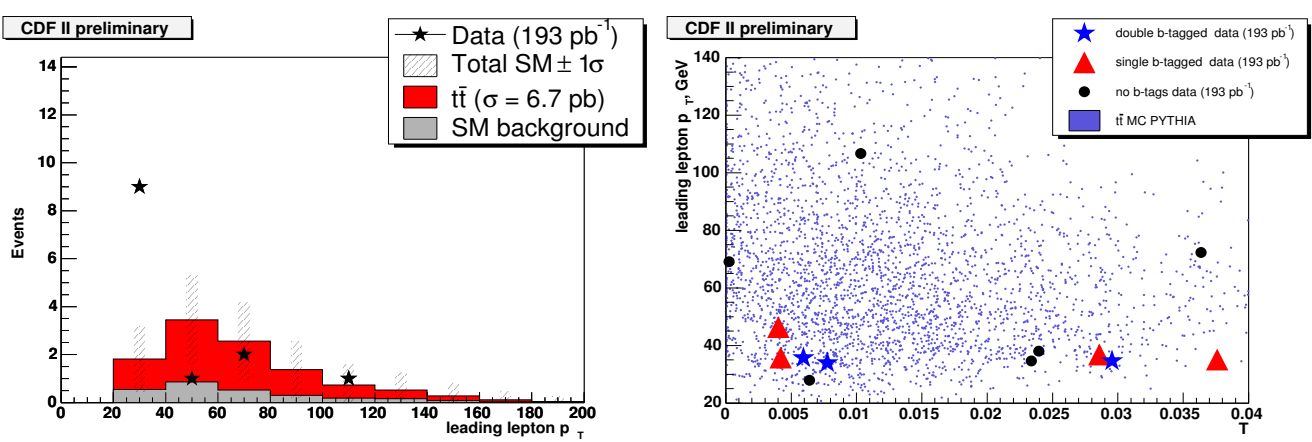

FIGURE 3. The leading lepton $p_{\mathrm{T}}$ distribution (left) and $v s$. the $T$ variable (right) with number of identified $b$-jets per event.

events each with a low weight if its kinematics depart from the standard model or a high weight if the kinematics are as expected. Next, a Kolmogorov-Smirnov test statistic is used to quantify the difference for each variable, by fitting shape distributions of the cumulative events in the subsample with the expected standard model events cumulative distribution.

This technique has been validated using large $W+$ jets data samples to assess how well we model events with dilepton characteristics. The data reproduces extremely well the simulation in the four concerned variables. A pass of the algorithm gives back high values of compatibility with the standard model, as expected.

Figure 3 shows the $t \bar{t}$ dilepton events distribution of lepton $p_{\mathrm{T}}$ and the plane $\left(p_{\mathrm{T}}, T\right)$ with further $b$-tagged jets information. There is an obvious excess of events with low $p_{\mathrm{T}}$ leptons and this translates into a small compatibility with the expectation. By applying the technique outlined above, the entire data set is found to be the one less similar to the standard model with a P-value of $1.6 \%$. If systematic uncertainties are included, then the $\mathrm{P}$-value grows to $4.5 \%$. A careful examination of these 13 candidate events shows that most of them seem to be real top dilepton events, with high values of $T$ and at least one identified $b$ jet.

To summarize, the relative excess of low lepton $p_{\mathrm{T}}$ events in the dilepton sample can be attributed to a statistical fluctuation, but overall no kinematic anomalies are observed in the Run II data so far that confirm the Run I peculiarities.

\section{W HELICITY}

Neglecting the $b$ quark mass, top quarks are produced at the Tevatron as left-handed fermions, i.e. with the spin in opposite direction to the top quark direction of motion. By conservation of angular momentum - the (massless) $b$ quark is always left-handed- $W$ bosons can only be produced with longitudinal polarization (zero helicity: $W_{0}$ ) or with left-handed polarization (helicity -1: $W_{-}$). Top quark decays to right-handed $W_{+}$bosons are forbidden in the standard model. Moreover, the rate at which the top quark decays to 

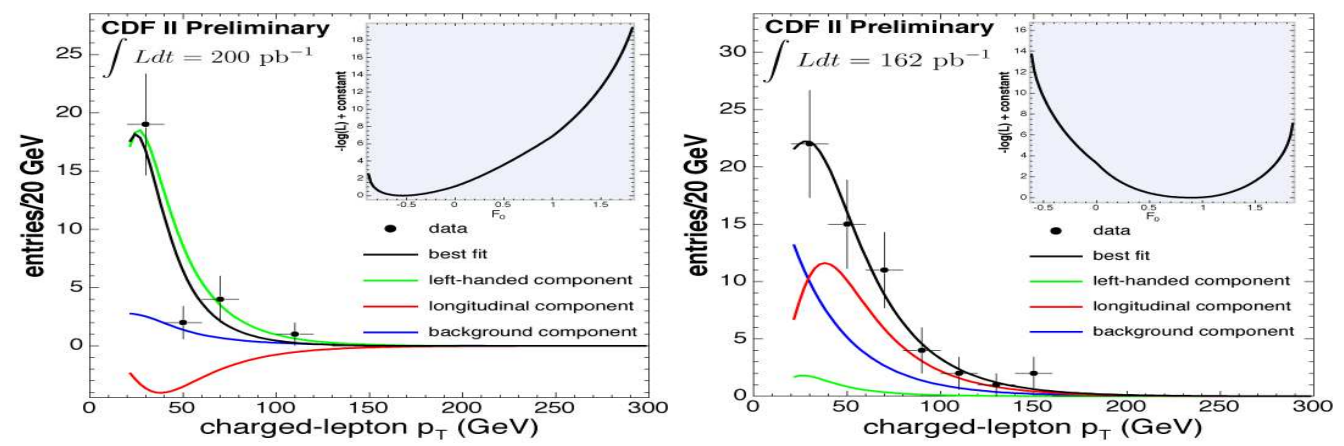

FIGURE 4. The lepton $p_{\mathrm{T}}$ distributions for the dilepton (left) and $\ell+$ jets (right) data samples, overlaid with the normalized signal and background PDFs. Inset is the $-\log \mathscr{L}$ as a function of $F_{0}$.

a longitudinally polarized $\mathrm{W}$ boson can be calculated in terms of $m_{t}$ and $m_{W}$ :

$$
F_{0} \equiv \frac{\Gamma\left(t \rightarrow W_{0} b\right)}{\Gamma\left(t \rightarrow W_{0} b\right)+\Gamma\left(t \rightarrow W_{T} b\right)}=\frac{1}{1+2\left(m_{W} / m_{t}\right)^{2}}=0.7 \quad\left(\text { for } m_{t}=175 \mathrm{GeV} / c^{2}\right)
$$

Thus, $F_{-}=0.3$ and $F_{+}=0$ in the standard model. There are several methods to measure $F_{0}$ and determine the helicity content of the $W$ bosons: one can measure an angular distribution (the lepton is emitted in a different direction depending on the $W$ polarization), or the lepton $p_{\mathrm{T}}$ spectrum (leptons from $W_{0}$ are emitted with more energy, in average, than those from $W_{-}$, and with less energy than those from $W_{+}$).

$\mathrm{CDF}$ has presented analyses with both approaches, a $\cos \theta^{*}$ template fit $\left(\theta^{*}\right.$ is the angle between the lepton in the $\mathrm{W}$ rest frame and the $\mathrm{W}$ momentum in the top rest frame) [12] and a lepton $p_{\mathrm{T}}$ spectrum fit [13]. DØ has performed a similar analysis in which a limit on $F_{+}$is calculated (instead of measuring $F_{0}$ ) by fitting to $\cos \theta^{*}$ distributions from non-zero $F_{+}$[14]. Here we describe briefly the lepton $p_{\mathrm{T}}$ spectrum fit from CDF.

Using the dilepton $\left(161 \mathrm{pb}^{-1}\right)$ and $\ell+$ jets $\left(193 \mathrm{pb}^{-1}\right)$ samples and the SECVTX $b$ tagging algorithm for the latter, the analysis proceeds to build a likelihood function that includes the PDFs of $p_{\mathrm{T}}$ of leptons from background and signal samples for the different helicities $\left(0\right.$ and -1 , assuming $\left.F_{+}=0\right)$.

Figure 4 presents the lepton $p_{\mathrm{T}}$ spectrum for the dilepton and $\ell+$ jets data. As was seen in the previous section, the dilepton analysis has selected softer candidate events than expected. This forces the longitudinal component to be negative to be able to fit the data. The minimum of $-\log \mathscr{L}$ appears at $F_{0}=-0.54$, which is an unphysical value and inconsistent with $F_{0}=0.70$ at the $\sim 2 \sigma$ level. In the $\ell+$ jets analysis, we obtain a physical result of: $F_{0}=0.88_{-0.47}^{+0.12}$. The dilepton result is consistent with the $\ell+$ jets result at the $2 \sigma$ level, so a combined result yields: $F_{0}=0.27_{-0.21}^{+0.35}$. This is inconsistent with the standard model at the $1 \sigma$ level. 


\section{SPIN CORRELATIONS}

Tevatron collisions produce unpolarized top and anti-top quarks. But the spins of these pairs are correlated. To measure the spin polarization of the parent top quarks, experimentally it is more effective to use the $W$ boson decays to leptons and not to quarks. By measuring the production angle of the two leptons one can measure the spin correlation between the two parent top quarks. That is, the spin correlation of the top quarks can be measured by the angular correlation of the decay products:

$$
\frac{1}{\sigma} \frac{d^{2} \sigma}{d\left(\cos \theta_{+}\right) d\left(\cos \theta_{-}\right)}=\frac{1+\kappa \cos \theta_{+} \cdot \cos \theta_{-}}{4}
$$

where $\kappa / 4=\left(N_{\uparrow \uparrow}-N_{\uparrow \downarrow}\right) /\left(N_{\uparrow \uparrow}+N_{\uparrow \downarrow}\right)$ is the correlation coefficient, and $N_{\uparrow \uparrow}$ is the number of events with top quark spins aligned $\left(\cos \theta_{+} \cdot \cos \theta_{-}>0\right)$ and $N_{\uparrow \downarrow}$ is the number of events with top quark spins in opposite directions $\left(\cos \theta_{+} \cdot \cos \theta_{-}<0\right) ; \theta_{ \pm}$is the angle between one of the charged leptons ( + or - ) and the axis of quantization in the rest frame of its parent top quark. The spin basis most effective to measure this asymmetry at the Tevatron is the so called off-diagonal basis, which defines the axis of quantization as a function of the velocity $\beta^{*}$ and the scattering angle $\theta^{*}$ of the top quark with respect to the incoming partons:

$$
\tan \psi=\frac{\beta^{* 2} \sin \theta^{*} \cos \theta^{*}}{1-\beta^{* 2} \sin ^{2} \theta^{*}}
$$

where $\psi$ is the angle between the spin basis axis and the $p \bar{p}$ beam axis.

Measuring this correlation coefficient is interesting for three reasons: it is a proof that the top quark behaves like a free quark, unaffected by confinement effects; its observation would yield an indirect lower bound on $\left|V_{t b}\right|$, without the assumption of unitarity, because the top quark lifetime depends on $\left|V_{t b}\right|$; and finally, new physics with different top quark dynamics could modify this correlation.

The DØ collaboration performed this measurement in Run I with $125 \mathrm{pb}^{-1}$ of dilepton data (including electrons and muons) [15]. The dilepton channel has the advantage of clean lepton identification, smaller background, and easier association of final objects with the initial originating quarks. But the final sample is small and two neutrinos must be reconstructed from the missing energy in the event. We therefore have six unknowns (the momenta of the two neutrinos) and five constraints $\left(m\left(\ell_{1} v_{1}\right)=m\left(\ell_{2} v_{2}\right)=m_{W}\right.$, and $\left.m\left(\ell_{1} v_{1} b_{1}\right)=m\left(\ell_{2} v_{2} b_{2}\right)\right)$. The problem can be solved if we assume $m\left(\ell_{i} v_{i} b_{i}\right)=m_{t}=$ $175 \mathrm{GeV} / c^{2}$. The system is then solved for the neutrino momentum vectors and each of the possible four solutions is assigned a weight based on the difference between the sum of $p_{\mathrm{T}}$ of the two neutrinos in the solution and the measured $\mathbb{E}_{\mathrm{T}}$ in the event. An event fit is then performed to the $t \bar{t}$ hypothesis by boosting the final objects to the frame of the original top quarks. Out of the many solutions of the fit, a two-dimensional binned likelihood analysis is performed on the plane $\left(\cos \theta_{+}, \cos \theta_{-}\right)$. By comparing Monte Carlo and data, the final result is extracted: $\kappa>-0.25$ at $68 \%$ C.L., which is 
in agreement with the standard model prediction $\kappa=0.88\left({ }^{2}\right)$. For Run II we expect to have an increased sensitivity and possibly obtain an observation of $\kappa$ at the $2 \sigma$ level by including the $\ell+$ jets channel and a larger data sample.

\section{RESONANCE PRODUCTION}

Another interesting aspect of top quark physics is its production mechanism. At the Tevatron, the dominant production is through gluon exchange $(q \bar{q} \rightarrow t \bar{t}, g \bar{g} \rightarrow t \bar{t})$, but electroweak production of top quarks, via the interchange of a $W$ boson $\left(q g \rightarrow q^{\prime} t b\right.$ and $\left.q q^{\prime} \rightarrow t b\right)$ is also possible and actively looked for by both collaborations. Additionally, some models of electroweak symmetry breaking, like top-color assisted technicolor, predict new heavy $Z^{\prime}$ bosons that couple to the third generation of quarks and not to leptons, giving $Z^{\prime} \rightarrow t \bar{t}$. Other possibility are narrow resonances (compared to the detector resolution) of a heavy top quark condensate: $X \rightarrow t \bar{t}$. The $\mathrm{D} \varnothing$ collaboration published an analysis over $130 \mathrm{pb}^{-1}$ of Run I data in search for this non-standard model production of top quark pairs [16].

The analysis uses the $\ell+$ jets topology and selection as in the top quark mass calculation [17] with two orthogonal analyses, one with topological cuts and the other with soft muon tagging (to tag $b$ jets). Events are fitted to a three-constrain (3C) hypothesis for $t \bar{t}$ production and decay, taking into account all possible permutations of jet assignment and longitudinal momentum of the neutrino, the minimum $\chi^{2}$ solution is used to reconstruct the invariant mass $M_{t \bar{t}}$. A total of 41 events pass the selection criteria, of which four are $\mu$ tagged. We expect $4.2 \pm 3.2$ from the signal $X \rightarrow t \bar{t}, 23.7 \pm 11.6$ from standard model $t \bar{t}$ production and $15.4 \pm 10.6$ from $W+$ jets and QCD background.

Since data and background expectation are compatible, a Bayesian upper limit is calculated on the $\sigma_{X} B(X \rightarrow t \bar{t})$ at the $95 \%$ C.L. for $M_{X}$ between 400 and $1000 \mathrm{GeV} / c^{2}$ ranging from $5.0 \mathrm{pb}$ to $2.0 \mathrm{pb}$, respectively. $Z^{\prime}$ bosons, as predicted in technicolor models with $\Gamma_{X}=0.012 M_{X}$ are excluded with masses $M_{X}<560 \mathrm{GeV} / c^{2}$ at $95 \%$ C.L.

\section{CONCLUSIONS}

Both CDF and DØ are actively studying the top quark physics. We have presented here the most recent results that are not concerned with the mass or cross section measurements, but rather with the properties of the top quark decays and how top quarks may be produced at the Tevatron from new physics. Some aspects not mentioned here, like single top production which will provide direct access to $\left|V_{t b}\right|$, or searches for flavor changing neutral currents $(t \rightarrow q Z$ and $t \rightarrow q \gamma$ with $q=u, c$ are expected to be extremely rare) are also possible amongst many other interesting studies. The Tevatron offers an excellent opportunity to further our understanding on the very special nature of top

\footnotetext{
2 The off-diagonal basis is constructed to maximize the spin asymmetry in $t \bar{t}$ events at the small relativistic energies of the Tevatron. In this basis, $\kappa=1$ for $q \bar{q} \rightarrow t \bar{t}$, but adding the process $g g \rightarrow t \bar{t}$ brings down the standard model value to $\kappa=0.88$
} 
quarks. From continuing our precision measurements on standard model parameters to exciting new discoveries of new phenomena with at least $2 \mathrm{fb}^{-1}$ by 2007, or some 1000 $t \bar{t}$ events per experiment.

\section{ACKNOWLEDGMENTS}

It is a pleasure to congratulate the organizers of Hadron Collider Physics 2004 for a pleasant and successful conference at Michigan State University. The top group conveners from DØ, Aurelio Juste and Arnulf Quadt, and CDF, Jaco Konigsberg and Evelyn Thomson, were of great help in preparing this talk.

\section{REFERENCES}

1. F. Abe et al. [CDF Collaboration], "Observation of top quark production in anti-p p collisions," Phys. Rev. Lett. 74, 2626 (1995) [arXiv:hep-ex/9503002] ; S. Abachi et al. [D0 Collaboration], "Observation of the top quark," Phys. Rev. Lett. 74, 2632 (1995) [arXiv:hep-ex/9503003].

2. P. Azzi et al., "Combination of CDF and D0 results on the top-quark mass," [arXiv:hep-ex/0404010], http: //tevewwg. fnal.gov/

3. The latest experimental results in top quark physics can be accessed at: http://www-cdf.fnal.gov/physics/new/top/top.html, http://wWw-d0.fnal.gov/Run2Physics/WWW/results/TOP/top.htm

4. [CDF Collaboration], "Measurement of $B(t \rightarrow W b) / B(t \rightarrow W q)$," CDF note 7172

5. G. J. Feldman and R. D. Cousins, "A Unified approach to the classical statistical analysis of small signals,” Phys. Rev. D 57, 3873 (1998) [arXiv:physics/9711021].

6. T. Affolder et al. [CDF Collaboration], "First measurement of the ratio $\mathrm{B}(\mathrm{t} \rightarrow \mathrm{W}$ b)/B(t $\rightarrow \mathrm{W}$ q) and associated limit on the CKM element $|\mathrm{V}(\mathrm{tb})|$," Phys. Rev. Lett. 86, 3233 (2001) [arXiv:hepex/0012029].

7. [CDF Collaboration], "A measurement of $B(t \rightarrow \tau v q)$," CDF note 7179

8. F. Abe et al. [CDF Collaboration], "Measurement of the top quark mass and t anti-t production cross section from dilepton events at the Collider Detector at Fermilab," Phys. Rev. Lett. 80, 2779 (1998) [arXiv:hep-ex/9802017].

9. [CDF Collaboration], "Search for anomalous kinematics in $t \bar{t}$ dilepton events at CDF," CDF note 7114

10. R. M. Barnett and L. J. Hall, “A multilepton signal for supersymmetric particles in Tevatron data?," Phys. Rev. Lett. 77, 3506 (1996) [arXiv:hep-ph/9607342].

11. D. Acosta et al. [CDF Collaboration], "Measurement of the $\mathrm{t}$ anti-t production cross section in $\mathrm{p}$ anti-p collisions at $\mathrm{s}^{* *}(1 / 2)=1.96-\mathrm{TeV}$ using dilepton events," [arXiv:hep-ex/0404036].

12. [CDF Collaboration], "Measurement of $\mathrm{W}$ boson polarization in top quark decays using $\cos \theta^{*}$ at CDF II," CDF note 7173

13. [CDF Collaboration], "A mesurement of the fraction of longitudinally-polarized W bosons produced in top-quark decays in $200 \mathrm{pb}^{-1}$ of $p \bar{p}$ collisions at $\sqrt{s}=1.96 \mathrm{TeV}$," CDF note 7058

14. [D0 Collaboration], "Measurement of the W helicity in top quark decays," D0 note 4549

15. B. Abbott et al. [D0 Collaboration], "Spin correlation in $\mathrm{t}$ anti-t production from $\mathrm{p}$ anti-p collisions at $s^{* *}(1 / 2)=1.8-T e V$,” Phys. Rev. Lett. 85, 256 (2000) [arXiv:hep-ex/0002058].

16. V. M. Abazov et al. [D0 Collaboration], "Search for narrow t anti-t resonances in $\mathrm{p}$ anti-p collisions at $s^{* *}(1 / 2)=1.8-T e V$,” Phys. Rev. Lett. 92, 221801 (2004) [arXiv:hep-ex/0307079].

17. B. Abbott et al. [D0 Collaboration], "Direct measurement of the top quark mass at D0," Phys. Rev. D 58, 052001 (1998) [arXiv:hep-ex/9801025]. 